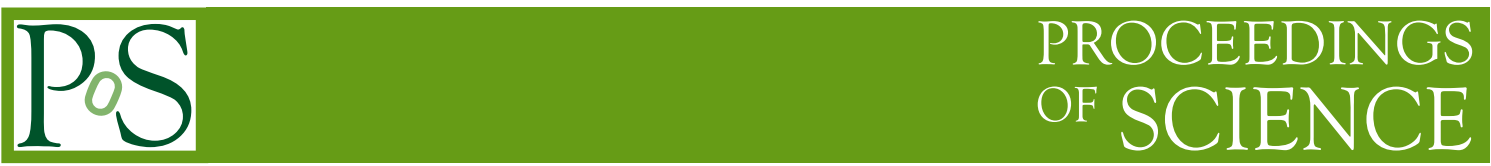

\title{
Galactic ${ }^{26} \mathrm{Al},{ }^{60} \mathrm{Fe}$ and ${ }^{60} \mathrm{Fe} /{ }^{26} \mathrm{Al} \gamma$-ray flux ratio from massive stars
}

\author{
Moisès Suades* \\ Institut de Ciències de l'Espai (CSIC-IEEC), Bellaterra (Barcelona), Spain \\ E-mail: suades@ieec.uab.es
}

\section{Margarita Hernanz}

Institut de Ciències de l'Espai (CSIC-IEEC), Bellaterra (Barcelona), Spain

E-mail: hernanz@ieec.uab.es

\section{Nicolas de Séréville}

Institut de Physique Nucléaire (IPN), Orsay, France

E-mail: deserevi@ipno.in2p3.fr

\begin{abstract}
The radioactive nucleus ${ }^{60} \mathrm{Fe}$ has been recently detected in the Galaxy for the first time, with the RHESSI (Reuven Ramaty High Energy Solar Spectroscopic Imager) satellite and with the SPI spectrometer onboard INTEGRAL (International Gamma-Ray Astrophysics Laboratory). In addition, galactic emission from ${ }^{26} \mathrm{Al}$ has been confirmed and observed with excellent energetic resolution with these two missions. In the light of these advances, an analysis of these two radioactive isotopes and the $\gamma$-ray line flux ratio between them is presented, under the assumption that massive stars are the sole contributors. We discuss the range of uncertainty of the yields derived from theoretical stellar models and focus on the influence of metallicity on them. Comparison between theoretical and observed ${ }^{60} \mathrm{Fe} /{ }^{26} \mathrm{Al}$ flux ratios is a potential tool to claim a global need of extra sources of ${ }^{26} \mathrm{Al}$, as well as to discard or support some prescriptions for the mass loss rate affecting the ejection of the synthesized ${ }^{26} \mathrm{Al}$ and ${ }^{60} \mathrm{Fe}$ into the interstellar medium. However, the broad range of theoretical ${ }^{60} \mathrm{Fe} /{ }^{26} \mathrm{Al}$ ratios obtained prevents to extract accurate constraints on theoretical models of massive stars.
\end{abstract}

Supernovae: lights in the darkness (XXIII Trobades Científiques de la Mediterrània) October 3-5 2007

Mao, Menorca, Spain

\footnotetext{
* Speaker.
} 


\section{Introduction}

Nucleosynthesis in stars and further mass ejection through supernova explosions are responsible for the enrichment of the Galaxy with new elements. ${ }^{26} \mathrm{Al}$ and ${ }^{60} \mathrm{Fe}$ are two radioactive isotopes synthesized in massive stars which decay emitting gamma-ray lines with respective lifetimes of 1 and 2 Myr. These lifetimes are long as compared with the time interval between supernova events, where both elements are assumed to be mainly produced, leading to their accumulation in the Galaxy. But at the same time these lifetimes are short as compared to the age of the Galaxy, and therefore the detection of ${ }^{26} \mathrm{Al}$ and ${ }^{60} \mathrm{Fe}$ emission lines proves that nucleosynthesis is an ongoing process in the Galaxy.

After the pioneering detection of the ${ }^{26} \mathrm{Al} \gamma$-ray emission line at $1.809 \mathrm{MeV}$ with the HEAO-3 satellite [12,13], other $\gamma$-ray instruments, either on balloon flights or onboard satellites, have corroborated this detection. The observations of COMPTEL instrument on board the CGRO satellite made the first all-sky map of the emission from galactic ${ }^{26} \mathrm{Al}$ possible [4], and its clumpy distribution along the plane of the Galaxy revealed that $1.809 \mathrm{MeV}$ photons mainly trace the massive star population. Four years later a good spatial correlation was shown between the COBE/DMR allsky map of the electronic free-free emission at $53 \mathrm{GHz}$ and the ${ }^{26} \mathrm{Al}$ map from COMPTEL [8, 9]. This was another evidence in favour of massive stars as the main contributors to ${ }^{26} \mathrm{Al}$, since 53 $\mathrm{GHz}$ emission traces regions of ionized gas, typically around massive stars. However, a moderate contribution from AGB stars and classical novae cannot be ruled out from the data, before better spatial coverage is available.

The long awaited detection of the ${ }^{60} \mathrm{Fe}$ characteristic $\gamma$-ray lines at 1.173 and $1.332 \mathrm{MeV}$ has been possible thanks to RHESSI and INTEGRAL satellites, which have also confirmed galactic ${ }^{26} \mathrm{Al}$ emission. Observations with both satellites have provided ${ }^{60} \mathrm{Fe} /{ }^{26} \mathrm{Al}$ line flux ratios in very good agreement with each other: $0.097 \pm 0.039$ with RHESSI [20], $0.11 \pm 0.03$ with INTEGRAL/SPI [6], and $0.148 \pm 0.06$ in a more recent analysis of a larger data set from SPI [22].

Radioactive ${ }^{60} \mathrm{Fe}$ is expected to be produced only in core collapse supernovae (cc SNe), which may also synthesize most of the galactic ${ }^{26} \mathrm{Al}$. With this assumption and adopting the yields of [24] (hereinafter WW95), a ${ }^{60} \mathrm{Fe} /{ }^{26} \mathrm{Al}$ flux ratio of 0.16 was predicted [21], in very good agreement with the recently observed value. However, updated models of nucleosynthesis in massive stars, such as [18] (hereinafter RHHW) and [2] (hereinafter CL04), predicted larger flux ratios. In a recent analysis [16], it has been claimed that a possible way to solve this apparent discrepancy is to include other contributors to the galactic ${ }^{26} \mathrm{Al}$, as the winds of Wolf-Rayet stars, an idea that has been also proposed in other papers [15, 7]. In addition, it is not yet clear how much the other potential ${ }^{26} \mathrm{Al}$ sources, i.e., AGB stars and classical novae, contribute to ${ }^{26} \mathrm{Al}$ [17]. The latest models of massive stars come back to smaller values of the flux ratio, in closer agreement again with the observations $[11,23]$. It is worth mentioning that [11] (hereinafter LC06) include the contribution of the WR star winds in their computation of the ${ }^{26} \mathrm{Al}$ and ${ }^{60} \mathrm{Fe}$ yields.

In this paper we present a consistent way of determining the theoretical ${ }^{60} \mathrm{Fe} /{ }^{26} \mathrm{Al}$ flux ratio derived 
from massive star population, paying special attention to the dependence of the stellar productivity of ${ }^{26} \mathrm{Al}$ and ${ }^{60} \mathrm{Fe}$ on metallicity. A comparison of our determinations with previous theoretical results and observations is made.

\section{2. ${ }^{60} \mathrm{Fe} /{ }^{26} \mathrm{Al}$ flux ratio determination}

As stressed by Timmes et al. [21], ${ }^{26} \mathrm{Al}$ and ${ }^{60} \mathrm{Fe}$ are mainly produced in massive stars and should then have a similar spatial distribution in the Galaxy; this has important implications on the observed ${ }^{60} \mathrm{Fe} /{ }^{26} \mathrm{Al} \gamma$-ray flux ratio. Therefore, we will focus our interest in massive stars in order to derive the theoretical flux ratio. Then the mean amount of one particular species $i$ created by a given population of stars in the mass range $\left[M_{l o w}, M_{\mathrm{up}}\right]$ becomes:

$$
\bar{Y}_{i}=\frac{\int_{M_{\text {low }}}^{M_{\text {up }}} Y_{i}(M) \phi(M) d M}{\int_{M_{\text {low }}}^{M_{\text {up }}} \phi(M) d M}
$$

where we average the yields $Y_{i}(M)$ by the initial mass function (IMF) of Salpeter [19]. Since the flux of a particular species is proportional to the number of nuclei, i. e., to the ratio of the averaged yield to its mass number, $F_{i}=\bar{Y}_{i} / A_{i}$, the flux ratio $\mathrm{FR}$ of ${ }^{60} \mathrm{Fe}$ to ${ }^{26} \mathrm{Al}$ turns to be ${ }^{1}$ :

$$
F R=\frac{26}{60} \frac{\int_{M_{\text {low }}}^{M_{\text {up }}} Y_{60}(M) \phi(M) d M}{\int_{M_{\text {low }}}^{M_{\text {up }}} Y_{26}(M) \phi(M) d M}
$$

We consider four different possibilities for the yields: WW95, RHHW, CL04 and LC06. The first data set is the oldest, but the most complete one, since the authors take a range of masses between $11 M_{\odot}$ and $40 M_{\odot}$ and metallicities up to solar value; it is still often taken as a reference. The second set of yields was computed by the same group and code, but with some improvements in stellar aspects (inclusion of mass loss related to stellar winds) and updated input physics (opacities, nuclear reaction network and rates). The third and fourth sets come from the same authors, but LC06 has improved physics (convective mixing and nuclear burning are solved simultaneously) and revised nuclear reaction rates. Although LC06 models are only for solar metallicity $(Z=0.02)$, a wide range of progenitor mass stars allowed them to include the Wolf-Rayet (WR) phase for stars up to $120 M_{\odot}$.

\subsection{Solar metallicity approximation}

As a first approximation, we have determined mean yields and flux ratios using data for solar metallicity (see Table 1) adopting the most recent data set LC06 and, for the sake of completeness, also the other three works. With WW95 yields (in their mass range from 12 to $40 M_{\odot}$ ), we have obtained a FR of 0.19; this value is in very good agreement with Timmes et al. [21], who used the same input data and determined a flux ratio of 0.16 from the integration along the galactic plane of the injection rates of ${ }^{26} \mathrm{Al}$ and ${ }^{60} \mathrm{Fe}$, computed with a code of galactic chemical evolution. These

\footnotetext{
${ }^{1}$ It is worth to mention that in his analysis, Prantzos [16] adopts a different approach, taking the IMF-averaged ratio of the yields, instead of the ratio of the IMF-averaged yields, which in our opinion is not a tracer of the flux ratio (e.g., his units for the FR are not correct). In addition, his FR should be monotonically increasing with $M_{\text {up }}$, and this is not the case, according to his Figure 2.
} 


\begin{tabular}{ccccc}
\hline Authors & $M_{\text {low }}-M_{\text {up }}\left(M_{\odot}\right)$ & $\bar{Y}_{26}$ & $\bar{Y}_{60}$ & FR \\
\hline WW95 & $12-40$ & 8.48 & 3.80 & 0.19 \\
RHHW & $13-25$ & 3.73 & 7.71 & 0.90 \\
CL04 & $13-35$ & 1.49 & 4.68 & 1.36 \\
LC06 & $11-120$ & 9.75 & 6.90 & 0.31 \\
\hline
\end{tabular}

Table 1: ${ }^{26} \mathrm{Al}$ and ${ }^{60} \mathrm{Fe}$ mean yields and ${ }^{60} \mathrm{Fe} /{ }^{26} \mathrm{Al}$ flux ratio adopting solar metallicity at birth for all masses. Units of $\bar{Y}_{26,60}: 10^{-5} M_{\odot}$.

are two independent ways to obtain the flux ratio, giving similar results; therefore, the method proposed here is robust and consistent with previous work. As can be seen in Figure 1, the flux ratio for LC06 yields increases with $M_{\text {up }}$, since ${ }^{60} \mathrm{Fe}$ yields increase more steeply with mass than ${ }^{26} \mathrm{Al}$ ones, in contrast with the WW95 case. We obtain that the FRs for CL04 and LC06 yields with $M_{\text {up }}=35 M_{\odot}$ are different (1.36 and 0.14 respectively, see Table 1 and Figure 1): according to the authors (see LC06 paper), their ${ }^{26} \mathrm{Al}$ yields in CL04 should be totally disregarded, because the ${ }^{26} \mathrm{Al}(\mathrm{p}, \gamma){ }^{27} \mathrm{Si}$ cross section they took from the REACLIB data base was wrong. Therefore, due to the unreliability of the results of CL04 and the small range of masses considered in RHHW, only WW95 and LC06 yields are displayed in Figure 1. In summary, the values obtained for FRs (Table 1) are well above the observed flux ratio, except for WW95 data $\left(M_{\mathrm{up}}=40 M_{\odot}\right)$ and for LC06 with $M_{\text {up }} \leq 80 M_{\odot}$. Incidentally, the FR with the latest yields of LC06 is very similar to that obtained with WW95 yields, if the same $M_{\text {up }}=40 \mathrm{M}_{\odot}$ is adopted (see Figure 1). But this seems to be a mere coincidence.

Nevertheless, WW95 yields are not free of uncertainties, since the authors did not consider mass loss, the upper mass was unrealistically low and some of their key nuclear reactions rates have been superseeded (see [23] and Section 2.2 for further discussion). Despite this fact, this is a very complete series of yields and they are taken as a reference for comparison. The aforementioned increase of the FR obtained from LC06 yields as a function of $M_{\text {up }}$ has important implications, because one should adopt $M_{\text {up }}$ as large as possible. In this case there is clearly a problem, which can only be solved with a different IMF, that strongly cuts the influence of the most massive stars. From Equation 2.2 it is easily seen that the $\gamma$-ray line flux ratio is a function which may strongly depend on the slope of the IMF, a feature already shown in Figure 15 in LC06.

We compare our results with those of LC06 adopting the Salpeter's IMF, i.e., $\phi(M) \propto M^{-(1+\gamma)}$, with a range of slopes from 0.1 to 2.5 , around the original Salpeter's $\gamma$ value 1.35. The extreme values of $\gamma$ are not representative of the observed mass spectrum, but they are useful in order to explore the maximum range in which the flux ratio might spread over, and then compare with observations. In Figure 2 data displayed correspond to the ${ }^{60} \mathrm{Fe} /{ }^{26} \mathrm{Al}$ flux ratio obtained with LC06 set of yields with mass loss rate of Nuggis \& Lammers [14] as a function of the slope $\gamma$ of the IMF, for different $M_{\text {up }}$ (see also the corresponding curve in Figure 15 from LC06). Figure 2 shows that only some combinations of $M_{\text {up }}$ and $\gamma$ provide a good fit: while all values of $\gamma$ are possible for $M_{\text {up }}=60 M_{\odot}$, only $\gamma \gtrsim 1.6$ for $M_{\text {up }}=80 M_{\odot}$, and $\gamma \gtrsim 2.2$ for $M_{\text {up }}=120 M_{\odot}$ are suitable. Therefore, 


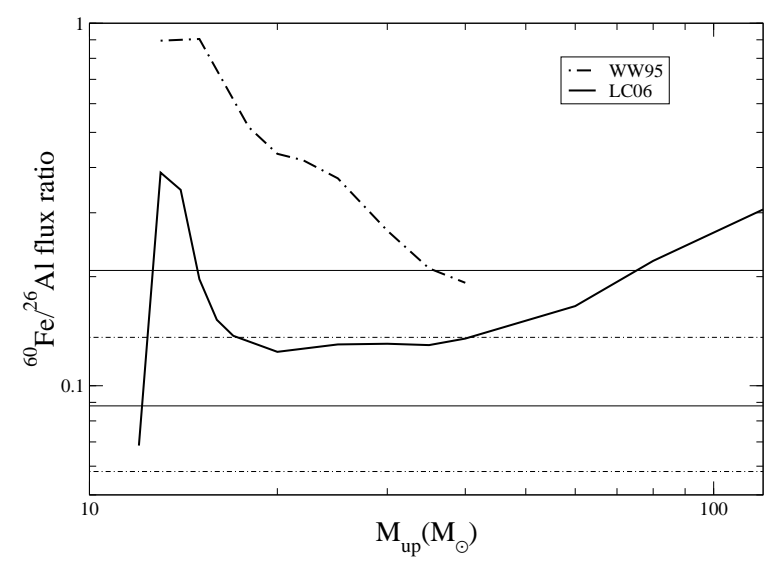

Figure 1: ${ }^{60} \mathrm{Fe} /{ }^{26} \mathrm{Al}$ flux ratio as a function of $M_{\text {up }}$ under the assumption that all stars have solar metallicity, for WW95 (dashed-dotted line) and LC06 (solid line) data sets. The horizontal bands in the lower part of the figure correspond to the observations of INTEGRAL (solid lines) and RHESSI (dashed-dotted lines).

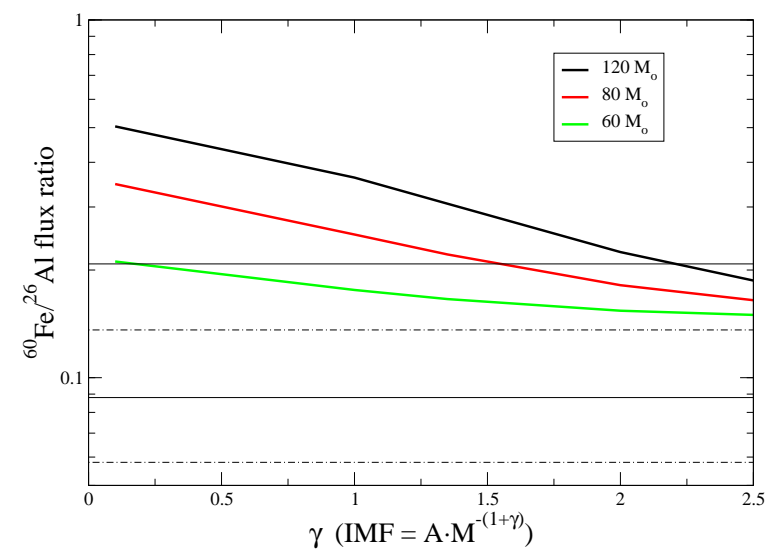

Figure 2: ${ }^{60} \mathrm{Fe} /{ }^{26} \mathrm{Al}$ flux ratio as a function of the IMF slope, $\gamma$, for different $M_{\text {up }}$, with yields extracted from LC06. The region between horizontal solid lines is the value of the flux ratio from INTEGRAL observations, whereas the region between horizontal dashed-dotted lines corresponds to results from RHESSI.

only in the case of $M_{\text {up }}=60 M_{\odot}$ it is possible to recover the observed FR with sensible values of $\gamma$, but this is an unrealistically low $M_{\text {up. }}$. On the other hand, not reliable large values of $\gamma(\sim 2.2)$ also provide observed FRs. It is worth mentioning that the best theoretical case would rather correspond to $M_{\text {up }}=120 M_{\odot}$ because of the inclusion of the winds of WR stars, but this particular case is the one which gives the largest FRs.

\subsection{The influence of initial metallicity}

${ }^{26} \mathrm{Al}$ and ${ }^{60} \mathrm{Fe}$ yields depend not only on the mass but also on the metallicity at birth of the star, which in turn depends on its location in the Galaxy (defined from its galactocentric radius $\mathrm{R}$ ): $Y_{26(60)}\left(M, Z_{\text {birth }}\right)=Y_{26(60)}(M, R)$. Since the lifetime of ${ }^{26} \mathrm{Al}$ and ${ }^{60} \mathrm{Fe}$ is much shorter than the age of the Galaxy $\left(t_{\mathrm{Gal}}\right)$, we should take into account stars exploding now as ccSNe and/or undergoing the WR phase, i.e., stars dying now. Furthermore, since we are dealing with massive stars, we can safely assume that their lifetimes are short as compared to $t_{\mathrm{Gal}}$, and thus their original metallicities 
are the current ones at their birth location. Therefore, a more careful analysis should take into account that massive stars, contributing to the current galactic content of ${ }^{26} \mathrm{Al}$ and ${ }^{60} \mathrm{Fe}$, were born with metallicities different than solar. For instance, in the inner Galaxy, where ${ }^{26} \mathrm{Al}$ and ${ }^{60} \mathrm{Fe}$ were first discovered, metallicities of massive stars should be much larger than solar.

We have used a code of chemical evolution of the Galaxy [3], based on the prescriptions of Alibés et al. [1], to determine the metallicity of stars born now at any galactocentric radius. The model is valid for the galactic disk and reproduces the observed age-metallicity relation in the solar neighborhood and the metallicity distribution of G-dwarf stars, the usual tests for this kind of codes. Assuming $t_{\mathrm{Gal}}=13 \mathrm{Gyr}$, we have found the metallicity of the Milky Way at two different radii: 0.036 at $2.5 \mathrm{kpc}$, representative of the central Galaxy (but out of the bulge), and 0.022 at 8.5 $\mathrm{kpc}$, representative of the solar neighbourhood. These metallicities are larger than solar and well inside the range of observed values.

There has been a large effort in the last years in computing yields for low initial metallicites, down to $\mathrm{Z}=0$, with the aim of interpreting observed abundances in very low-metallicity stars. However, there has not been a parallel effort to compute yields for stars with metallicites larger than solar, which are crucial for a correct evaluation of the ${ }^{26} \mathrm{Al}$ and ${ }^{60} \mathrm{Fe}$ content in the Galaxy. The lack of yields for $Z>Z_{\odot}$ has obligued us to adopt a theoretical prescription to obtain yields at large metallicities. The simplest way to proceed is to take the power law suggested by Prantzos [16], as well as by Palacios et al. [15]: $Y_{26(60)}=Y_{26(60), \odot} \cdot\left(\frac{Z}{Z_{\odot}}\right)^{\beta_{26(60)}}$, with $\beta_{26}=0.7$ and $\beta_{60}=2.0$. This is a first approximation guess, since yields strongly depend on mass loss and on the size of the convective core, both being influenced in a non linear way by metallicity (see LC06). As long as the computation of presupernova models and explosions does not provide realistic quantitative determination of the yields for large metallicities, our particular purpose is to obtain orientative values for these quantities in order to determine the range in which flux ratio can spread over. It is worth noticing that, either by chance or not, yields really follow a power law tendency for metallicities smaller than solar, and this is the metallicity range we have used to extract the values of $\beta_{26,60}$.

A summary of the flux ratios obtained for a range of metallicities (related to different positions in the Galaxy) and slopes of the IMF is displayed in Figure 3 only for the cases of LC06 and WW95. The values are larger than those computed with solar metallicities, because the increase with metallicity of the ${ }^{60} \mathrm{Fe}$ yields is larger than that of ${ }^{26} \mathrm{Al}$. At regions close to the galactic center, the FR is twice as large as that with solar metallicity; therefore, the disagreement between theory and observations becomes more severe than in the solar case. In order to understand how each set behaves with a changing slope of the IMF, we should refer to Figure 1, where it is shown that the flux ratio grows with $M_{\text {up }}$ for LC06 data, but decreases with it for WW95 data. Then, as an increasing $\gamma$ reduces the contribution of massive stars, the flux ratio of LC06 decreases as $\gamma$ increases. Instead, for WW95 this trend is reversed, i.e., larger values of $\gamma$ give larger flux ratios. This result is displayed in Figure 3 with the labels on top of the boxes. The horizontal dashes indicate the flux ratio when $\gamma=1.35$. 


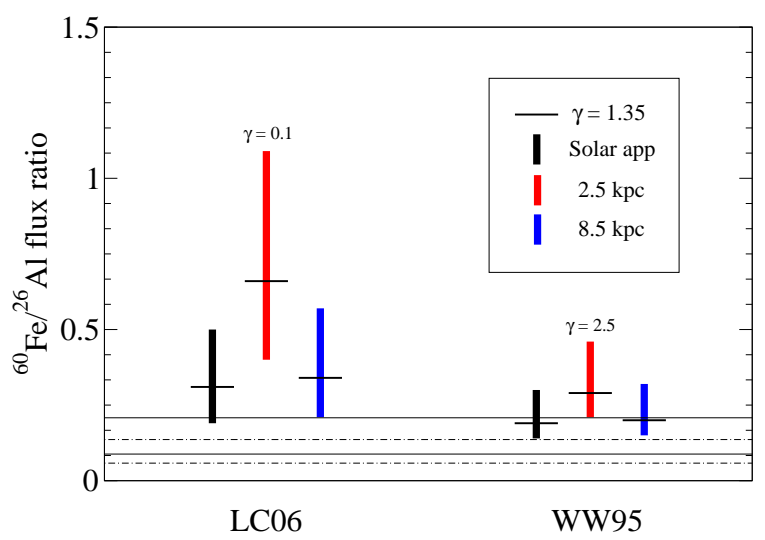

Figure 3: Range of validity of the ${ }^{60} \mathrm{Fe} /{ }^{26} \mathrm{Al}$ flux ratio by means of varying $\gamma$. The horizontal black dashes refer to the flux ratio determined by $\gamma=1.35$, and the labels at the top of each box refer to the value of $\gamma$ giving the upper limit of the flux ratio.

WW95 results are the closest to observations, but their nuclear network has been improved concerning the production of both ${ }^{26} \mathrm{Al}$ and ${ }^{60} \mathrm{Fe}$. As mentioned by Woosley \& Heger [23], new decay rates of the two main vias of destruction of ${ }^{26} \mathrm{Al}$ are up to 5 times greater than those used in WW95. Regarding ${ }^{60} \mathrm{Fe}$, the two nuclear rections ${ }^{59} \mathrm{Fe}(\mathrm{n}, \gamma)$ and ${ }^{60} \mathrm{Fe}(\mathrm{n}, \gamma)$ changed in RHHW in such a direction as to increase the production of this isotope; according to Woosley \& Heger [23], a rate for the former twice as large and a rate for the latter half as large than in WW95 would produce a flux ratio roughly twice larger. On the other hand, LC06 provide, in principle, the best yields since the problems in nuclear reactions are solved and they include the contribution of WR winds. However, the agreement between LC06's theoretical values and observations are far from being satisfactory, once the effect of metallicity at birth is included. A flux ratio of 0.66 is obtained, even more distant from the observed value than the previous determination, since the yields of LC06 present an enhancement of ${ }^{60} \mathrm{Fe}$ production. As stated in LC06, mass loss rate is still one of the main uncertainties concerning the evolution of a star. The fact that the authors adopt Nuggis \& Lammers [14] mass loss recipe for the WNE+WNO phases may drive an excessive ejection of ${ }^{60} \mathrm{Fe}$, and therefore unexpected high amounts of this isotope in the ISM are obtained. As a consequence, the flux ratio turns out to be too large.

\section{Summary and conclusions}

A simple prescription for the determination of the theoretical ${ }^{60} \mathrm{Fe} /{ }^{26} \mathrm{Al}$ gamma-ray line flux ratio has been derived, based on the definition of mean yield for a given stellar population. We have checked that this recipe gives results fully consistent with previous theoretical FR determinations based on the radial integration of the mass injection rate of ${ }^{26} \mathrm{Al}$ and ${ }^{60} \mathrm{Fe}$ along the Galaxy [21], when only massive stars are considered and the same set of yields (WW95, for solar metallicity) is adopted. On the contrary, our prescription does not agree with that of Prantzos [16] and does not give consistent results with it when the same yields are adopted.

We emphasize that theoretical values of the flux ratio depend on the metallicity at birth of stars 
ejecting the galactic radioactive nuclei ${ }^{26} \mathrm{Al}$ and ${ }^{60} \mathrm{Fe}$ into the ISM. This effect translates into a radial dependence of the FR, related to the current (if massive stars are the main contributors) metallicity distribution in the Galaxy. The central galactic regions should be considered as more representative of the observations, since the exposure time there with RHESSI and INTEGRAL is enhanced. The galactic metallicity gradient has been computed with a code of chemical evolution and the yields have been inferred following the suggestions of Prantzos [16] and Palacios et al. [15]. The derived theoretical FRs in the central Galaxy are much larger than observed, and only extremely large IMF slopes and/or small $M_{\text {up }}$ can alleviate the discrepancy.

It might exist some sign favouring the need of either extra sources of ${ }^{26} \mathrm{Al}$ apart from massive stars or stronger mass loss rates in order to enhance the production of ${ }^{26} \mathrm{Al}$ with respect to the ${ }^{60} \mathrm{Fe}$ one and thus reproduce the observed flux ratio more faithfully. Nevertheless, it is clear that accurate theoretical predictions, and thus accurate comparison between observations and models, can not be done without a good knowledge of the yields at $\mathrm{Z}>\mathrm{Z}_{\odot}$, since these are the most adequate for the population of massive stars; this is specially true in the central part of the Milky Way, where most of the ${ }^{26} \mathrm{Al}$ and ${ }^{60} \mathrm{Fe}$ has been detected with RHESSI and INTEGRAL. Only a better knowledge of the radial dependence of the observed flux ratio, together with a good knowledge of the yields at metallicities larger than solar, will permit to do a proper comparison between theory and observations based on the recipe presented here, and use it to put constraints on the models.

\section{Acknowledgments}

This research has been funded by MEC ESP2007-61593, AGAUR 2005-SGR00378, FEDER funds, and the MEC FPI fellowship BES-2005-9077.

\section{References}

[1] A. Alibés, J. Labay, R. Canal, Chemical evolution and abundance gradients in the Milky Way, 2001, A\&A, 370, 1103

[2] A. Chieffi, M. Limongi, Explosive yields of massive stars from $Z=0$ to $Z=Z_{\odot}, 2004$, ApJ, 608, 405

[3] N. de Séréville, Code of galactic chemical evolution (unpublished)

[4] R. Diehl et al., COMPTEL observations of galactic ${ }^{26}$ Al emission, 1995, A\&A, 298, 445

[5] R. Diehl et al., Radioactive ${ }^{26}$ Al from massive stars in the Galaxy, 2006, Nature, 439, 45

[6] M. J. Harris et al., Detection of $\gamma$-ray lines from interstellar ${ }^{60} \mathrm{Fe}$ by the high resolution spectrometer SPI, 2005, A\&A, 433, L49

[7] M. Hernanz, M. Suades, N. de Séréville, G. Martínez-Pinedo, J. José, in proceedings of the International Symposium on Nuclear Astrophysics Nuclei in the Cosmos IX, 2006, POS (NIC-IX) 115

[8] J. Knoedlseder, Implications of $1.8 \mathrm{MeV}$ gamma ray observations for the origin of ${ }^{26} \mathrm{Al}, 1999$, ApJ, 510,915

[9] J. Knoedlseder et al., A multiwavelength comparison of COMPTEL $1.8 \mathrm{MeV}{ }^{26} \mathrm{Al}$ line data, 1999, A\&A, 344, 68 
[10] N. Langer, Mass-dependent mass loss rates of Wolf Rayet stars, 1989, A\&A, 220, 135

[11] M. Limongi, A. Chieffi, The nucleosynthesis of ${ }^{26} \mathrm{Al}$ and ${ }^{60} \mathrm{Fe}$ in solar metallicity stars extending in mass from 11 to $120 M_{\odot}$ : the hydrostatic and explosive contributions, 2006, 647, 483

[12] W. A. Mahoney, J. C. Ling, A. S. Jacobson, R. E. Lingenfelter, Diffuse galactic gamma-ray line emission from nucleosynthetic ${ }^{60} \mathrm{Fe},{ }^{26} \mathrm{Al}$, and ${ }^{22} \mathrm{Na}$ preliminary limits from HEAO 3, 1982, ApJ, 262, 742

[13] W. A. Mahoney, J. C. Ling, W. A. Wheaton, A. S. Jacobson, HEAO 3 discovery of ${ }^{26}$ Al in the interstellar medium, 1984, ApJ, 286, 578

[14] T. Nugis, H. J. G. L. M. Lamers, Mass loss rates of Wolf Rayet stars as a function of stellar parameters, 2000, A\&A, 360, 227

[15] A. Palacios et al., New estimates of the contribution of Wolf Rayet stellar winds to the galactic ${ }^{26} \mathrm{Al}$, 2005, A\&A, 429, 613

[16] N. Prantzos, Radioactive ${ }^{26} \mathrm{Al}$ and ${ }^{60} \mathrm{Fe}$ in the Milky Way: implications of the RHESSI detection of ${ }^{60} \mathrm{Fe}, 2004, \mathrm{~A} \& \mathrm{~A}, 420,1033$

[17] N. Prantzos, R. Diehl, Radioactive ${ }^{26}$ Al in the Galaxy: observations versus theory, 1996, Phys. Rep., 267,1

[18] T. Rauscher, A. Heger, R. D. Hoffman, S. E. Woosley, Nucleosynthesis in massive stars with improved nuclear and stellar physics, 2002, ApJ, 576, 323

[19] E. E. Salpeter, The luminosity function and stellar evolution, 1955, ApJ, 121, 161

[20] D. M. Smith, in proceedings of 5th INTEGRAL Workshop on the INTEGRAL Universe (ESA SP-552), Munich 2004

[21] F. X. Timmes et al., ${ }^{26}$ Al and ${ }^{60}$ Fe from supernova explosions, 1995, ApJ, 449, 204

[22] W. Wang et al., SPI observations of the diffuse ${ }^{60}$ Fe emission in the Galaxy, 2007, A\&A, 469, 1005

[23] S. E. Woosley, A. Heger, Nucleosynthesis and remnants in massive stars of solar metallicity, 2007, $\mathrm{PhR}, 442,269$

[24] S. E. Woosley, T. A. Weaver, The evolution and explosion of massive stars. II. Explosive hydrodynamics and nucleosynthesis, 1995, ApJ, 101, 181 